\title{
A NOTE ON INVARIANT INTEGRALS ON LOCALLY COMPACT SEMIGROUPS
}

\author{
L. N. ARGABRIGHT
}

1. Introduction. An integral on a locally compact (Hausdorff) semigroup $S$ is a nontrivial, positive, linear functional $I$ on the space $C(S)$ of continuous real-valued functions on $S$ with compact supports. If $S$ satisfies the condition

(\#) for each compact set $K \subset S$ and each element $a \in S$, the set

$$
K a^{-1}=\{x \in S \mid x a \in K\}
$$

is compact; then, whenever $f \in C(S)$ and $a \in S$, the function $f_{a}$ defined by $f_{a}(x)=f(x a)$ is also in $C(S)$.

In this case, an integral $I$ on $S$ is called right invariant provided $I(f)=I\left(f_{a}\right)$ for all $f \in C(S)$ and all $a \in S$. A regular Borel measure $\mu$ on $S$ is called $r^{*}$-invariant if $\mu\left(B a^{-1}\right)=\mu(B)$ for all Borel sets $B$ and all $a \in S$.

J. H. Michael [7] introduced the above concept of an invariant integral ${ }^{1}$ and proved that if $S$ contains a unique minimal left ideal and satisfies some additional conditions (see. [7]) then $S$ admits a right invariant integral. P. S. Mostert [8] then pointed out that Michael's conditions could be weakened considerably and he also gave a much shorter proof. In this note we prove the following:

THEOREM 1. Let $S$ be a locally compact semigroup satisfying the condition (\#). Then the following are equivalent.

(a) $S$ admits a right invariant integral.

(b) $S$ admits an $r^{*}$-invariant measure.

(c) $S$ contains a unique minimal left ideal (which is necessarily closed).

Thus we obtain a complete generalization of the theorem of W. G. Rosen [10] on the existence of invariant means on compact semigroups. In addition, we make some remarks concerning the existence and structure of $r^{*}$-invariant measures on semigroups which do not necessarily satisfy (\#).

For matters concerned with integrals and measures on locally compact spaces, we follow Bourbaki [2, Chapter III], and Hewitt and Ross [6, \$11]. In particular, the family of Borel sets is defined to be the $\sigma$-algebra generated by the family of all open sets.

Received by the editors April 27, 1965.

1 Our condition (\#) is a weaker form of Michael's condition (A). 
The author is indebted to Professor Samuel Bourne for several stimulating conversations related to the topics of this paper. This work was supported in part by the National Science Foundation, under grant NSF GP-2026.

2. Right-invariant integrals on locally compact left groups. In this section we establish the existence and analyze the structure of rightinvariant integrals on locally compact left groups. Recall that a left group is a semigroup $S$ which is left simple $(S a=S$ for all $a \in S)$ and right cancellative. It is well known (see $[4$, p. 38]) that the following conditions on a semigroup $S$ are equivalent:

(i) $S$ is a left group.

(ii) $S$ is left simple and contains an idempotent.

(iii) $S$ is isomorphic to a direct product $E \times G$ where $E$ is a left zero semigroup and $G$ is a group.

In regard to the latter, we recall that each idempotent acts as a right identity in $S$ and that a realization of $S$ as a direct product is accomplished by taking $E$ to be the set of all idempotents in $S$ and letting $G=e_{0} S$ where $e_{0}$ is a fixed (but arbitrary) element of $E$. The mapping $\left(e, e_{0} x\right) \rightarrow e x$ is then an isomorphism of $E \times G$ onto $S$. If, in addition, $S$ is a locally compact semigroup, then this mapping (call it $\phi$ ) is topological. In fact, we have the following structure theorem.

THEOREM 2. Let $S$ be a locally compact left group. Then $S$ is topologically isomorphic to a direct product $E \times G$ where $E$ is a locally compact left zero semigroup and $G$ is a locally compact topological group.

This theorem is a special case of the theorem proved by Mostert in [8]; we omit the proof.

Now we apply the above structure theorem to establish the existence and structure of right-invariant integrals on a locally compact left group $S$. Note that each element of $S$ has a right inverse with respect to some right identity in $S$ and hence the right translates $r_{a}(x)$ $=x a$ are homeomorphisms of $S$. Thus $S$ satisfies the condition (\#).

Theorem 3. Let $S$ be a locally compact left group. Then $S$ admits a right-invariant integral. Moreover, each right-invariant integral I on $S$ can be obtained as follows:

Write $S=E \times G$ as in Theorem 2. Then

$$
I(f)=\int_{S} f d(\nu \times \lambda)
$$

where $\nu$ is a positive regular Borel measure on $E$ and $\lambda$ is a right Haar measure on $G$. 
Proof. Writing (1) as an iterated integral, it is easy to see that any such measure gives rise to a right-invariant integral on $S$. See [1, Theorem 1.2], for the details of a similar argument.

Suppose, conversely, that $I$ is a right-invariant integral on $S$. We observe that $I$ is completely determined by its values on functions of the form $h(e, g)=\psi(e) \phi(g)$ where $\psi \in C(E)$ and $\phi \in C(G)$; this is because linear combinations of functions of this type are "riche" in $C(E \times G)$ (see [2, p. 55 and p. 89]). Choose fixed nonnegative functions $\psi_{0} \in C(E)$ and $\phi_{0} \in C(G)$ such that $I\left(\psi_{0} \phi_{0}\right)=1$, and let $H(\phi)$ $=I\left(\psi_{0} \phi\right)$ for all $\phi \in C(G)$. Then $H$ is a positive, right-invariant integral on $G$; that is, $H$ is a right Haar integral on $G$. Next we note that for each fixed $\psi \in C(E)$, the mapping $\phi \rightarrow I(\psi \phi)$ is a right-invariant Radon measure (see $[2$, p. 50$]$ ) on $G$. Hence, for each $\psi$, there exists a real number $J(\psi)$ such that

$$
I(\psi \phi)=J(\psi) H(\phi)
$$

for all $\phi \in C(G)$. It is easy to see that $J$ is an integral on $E$. Equation (2) also shows that $I$ is the product $J \times H$ of the integrals $J$ and $H$ (see [2, p. 89]). Finally, the formula (1) now follows from the wellknown relationship between integrals and measures.

We remark that the measure $\mu=\nu \times \lambda$ is both $r^{*}$-invariant and rightinvariant:

$$
\mu\left(B a^{-1}\right)=\mu(B)=\mu(B a) \text { for all Borel sets } B C S \text { and all } a \in S .
$$

3. Proof of Theorem 1. Throughout this section $S$ will denote a locally compact semigroup satisfying the condition (\#). It is convenient to introduce the following weak form of condition (b).

(b') $S$ admits a positive regular Borel measure $\mu$ satisfying $\mu\left(U a^{-1}\right)$ $=\mu(U)$ for all open sets $U \subset S$ and all $a \in S$. We will prove Theorem 1 by establishing the implications $(\mathrm{a}) \Rightarrow\left(\mathrm{b}^{\prime}\right) \Rightarrow(\mathrm{c}) \Rightarrow(\mathrm{a})$ and $(\mathrm{c}) \Rightarrow(\mathrm{b})$.

$(\mathrm{a}) \Rightarrow\left(\mathrm{b}^{\prime}\right)$. Let $I$ be a right-invariant integral on $S$ and let $\mu$ be the corresponding regular Borel measure on $S$. For open sets $U$, we have $\rho(U)=\sup \left\{I(f) \mid f \in C(S), 0 \leqq f \leqq \chi_{U}\right\}$; thus $\mu\left(U a^{-1}\right) \geqq$ $\sup \left\{I\left(f_{a}\right) \mid 0 \leqq f \leqq \chi_{U}\right\}=\mu(U)$.

Let $K$ be a compact set and $\epsilon>0$. By regularity of $\mu$, there exists an open set $U$ containing $K$ such that $\mu(U)<\mu(K)+\epsilon$. Since $S$ is locally compact, there exists a function $f \in C(S)$ such that $f: S \rightarrow[0,1]$, $f(K)=1, f(S \sim U)=0$. Then $\mu\left(K a^{-1}\right) \leqq \int_{S} f(t a) d \mu(t)=\int_{S} f(t) d \mu(t) \leqq \mu(U)$ $<\mu(K)+\epsilon$. Thus $\mu\left(K a^{-1}\right) \leqq \mu(K)$ for all $a \in S$. Note that if $K$ is compact then $K a$ is compact and, since $K \subset(K a) a^{-1}$, it follows that $\mu(K) \leqq \mu\left((K a) a^{-1}\right) \leqq \mu(K a)$.

Now let $U$ be open and let $K$ be a compact set contained in $U a^{-1}$. 
Then $K a$ is compact and contained in $U$, whence we have $\mu(K)$ $\leqq \mu(K a) \leqq \mu(U)$. But, again by regularity, $\mu\left(U a^{-1}\right)=\sup \{\mu(K) \mid K$ compact, $\left.K \subset U a^{-1}\right\}$, and it follows that $\mu\left(U a^{-1}\right) \leqq \mu(U)$. This completes the proof.

$\left(\mathrm{b}^{\prime}\right) \Rightarrow(\mathrm{c})$. Let $\mu$ be a measure on $S$ satisfying the condition $\left(\mathrm{b}^{\prime}\right)$, and let $F$ be the support of $\mu$. If $L$ is a nonempty closed left ideal in $S$ and $a \in L$, then $\mu(S \sim L)=\mu\left((S \sim L) a^{-1}\right)=\mu(\varnothing)=0$, whence it follows that $F \subset L$. Thus $L_{0}=\bigcap\{L \mid L$ is a closed left ideal $\}$ is nonempty and contains $F$. Clearly $L_{0}$ is the unique minimal closed left ideal in $S$. We will show that $L_{0} \subset L_{0} a$ for all $a \in S$, whence it follows that $L_{0}$ is the unique minimal (algebraic) left ideal.

Let $a \in S$ and $b \in L_{0}$. Since $L_{0} a$ is a left ideal we must have $L_{0} \subset \mathrm{Cl}\left(L_{0} a\right)$; thus there exists a net $\left\{t_{\alpha}\right\}$ in $L_{0}$ such that $\lim _{\alpha} t_{\alpha} a=b$. If $K$ is a compact neighborhood of $b$, then $\left\{t_{\alpha}\right\}$ is eventually in the compact set $K a^{-1} \cap L_{0}$, and hence there is a subnet $\left\{t_{\beta}\right\}$ which converges to a point $t_{0}$ in $L_{0}$. Then $t_{0} a=\lim _{\beta} t_{\beta} a=b$, and so $b \in L_{0} a$.

$\left(\right.$ c) $\Rightarrow$ (a) and (c) $\Rightarrow$ (b). Let $L_{0}$ be the unique minimal left ideal in $S$. We note that $L_{0} a$ is minimal for each $a \in S$ (see [3, Lemma 2.1]), and thus $L_{0} a=L_{0}$. In particular, $L_{0}$ is also a right ideal. We will show first that $L_{0}$ is closed, and then that $L_{0}$ is a left group.

Let $a \in S$ and $b \in \bar{L}_{0}$. Since $L_{0}=L_{0} a$, there is a net $\left\{t_{\alpha}\right\}$ in $L_{0}$ such that $t_{\alpha} a \rightarrow b$. If $K$ is a compact neighborhood of $b$, then $\left\{t_{\alpha}\right\}$ is eventually in $K a^{-1}$, and so there is a subnet $\left\{t_{\beta}\right\}$ which converges to some point $t_{0}$ in $\bar{L}_{0}$. Then $t_{0} a=\lim _{\beta} t_{\beta} a=b$, and so $b \in \bar{L}_{0} a$. We have shown that $\bar{L}_{0} a=\bar{L}_{0}$ for all $a \in S$. If $a \in L_{0}$, then we have $\bar{L}_{0}=\bar{L}_{0} a \subset L_{0}$; hence $L_{0}$ is closed. Now let $a_{0}$ be a fixed element of $L_{0}$. Then $\left\{t \in L_{0} \mid t a_{0}=a_{0}\right\}$ $=a a_{0}^{-1} \cap L_{0}$ is a nonempty, compact, subsemigroup of $L_{0}$ and hence contains at least one idempotent (see [9, Lemma 4]). Thus $L_{0}$ is a left group.

Now let $\sigma=\nu \times \lambda$ be a product measure on $L_{0}$ as in Theorem 3, and let $\mu(B)=\sigma\left(B \cap L_{0}\right)$ for all Borel sets $B \subset S$. Let $e$ be a fixed idempotent in $L_{0}$. Then, $e a \in L_{0}$ for each $a \in S$, and

$$
\begin{aligned}
B a^{-1} \cap L_{0} & =\left\{x \in L_{0} \mid x a \in B\right\}=\left\{x \in L_{0} \mid x(e a) \in B\right\} \\
& =\left\{x \in L_{0} \mid x(e a) \in B \cap L_{0}\right\}=\left(B \cap L_{0}\right)(e a)^{-1} \cap L_{0}
\end{aligned}
$$

for all $B C S$. Since $\sigma$ is $r^{*}$-invariant on $L_{0}$, it follows that

$$
\begin{aligned}
\mu\left(B a^{-1}\right) & =\sigma\left(B a^{-1} \cap L_{0}\right)=\sigma\left(\left(B \cap L_{0}\right)(a e)^{-1} \cap L_{0}\right) \\
& =\sigma\left(B \cap L_{0}\right)=\mu(B)
\end{aligned}
$$

for all Borel sets $B \subset S$. Similarly, for each $f \in C(S)$ we have 


$$
\begin{aligned}
\int_{S} f(x a) d \mu(x) & =\int_{L_{0}} f(x a) d \sigma(x)=\int_{L_{0}} f(x(e a)) d \sigma(x) \\
& =\int_{L_{0}} f(x) d \sigma(x)=\int_{S} f(x) d \mu(x) .
\end{aligned}
$$

Hence $I(f)=\int_{S} f d \mu$ is a right invariant integral on $S$.

4. Remarks on $r^{*}$-invariant measures. In this section $S$ will denote a locally compact semigroup not necessarily satisfying (\#). We note that if $S$ contains a closed right ideal $F$ which is a left group, then any product measure on $F$ of the type in Theorem 3 gives rise to an $r^{*}$-invariant measure on $S$. The author conjectures that every $r^{*}$ invariant measure arises in this way, and the remainder of this section is devoted to some observations which lend some support to this conjecture.

One can easily see (as in the proof of $\left(\mathrm{b}^{\prime}\right) \Rightarrow(\mathrm{c})$ ) that if $S$ admits an $r^{*}$-invariant measure $\mu$, then $S$ contains a unique minimal closed left ideal $L_{0}$ which must necessarily contain the support of $\mu$. Furthermore, the support of $\mu$ has the following tantalizing property.

Proposition 1. Suppose that $S$ admits an $r^{*}$-invariant measure $\mu$, and let $F$ be the support of $\mu$. Then $F$ is a closed right ideal in $S$ and $\mathrm{Cl}(F a)=F$ for all $a \in S$.

Proof. Let $a \in S, x \in F$, and let $U$ be an (open) neighborhood of $x a$. Then $x \in U a^{-1}$ and, since $x \in F$, it follows that $\mu(U)=\mu\left(U a^{-1}\right)>0$. Thus $x a \in F$, and so $F$ is a right ideal. Now let $a \in S, b \in F$, and let $V$ be an (open) neighborhood of $b$. Then $\mu\left(V a^{-1}\right)=\mu(V)>0$, and so $V a^{-1} \cap F \neq 0$. This implies that $V \cap F a \neq 0$, and we conclude that $b \in \mathrm{Cl}(F a)$.

We conjecture that $F$ must in fact be a left group and thus that $\mu$ is a measure of the type described above, but we have been able to prove this only in the case that $S$ is discrete.

Proposition 2. Let $S$ be a discrete semigroup. Then $S$ admits an $r^{*}$ invariant measure if and only if $S$ contains a right ideal which is a left group.

Proof. We have only to prove the necessity. Suppose then that $S$ admits an $r^{*}$-invariant measure $\mu$ and let $F$ be the support of $\mu$. By Proposition 1, we know that $F$ is a left simple right ideal. It remains only to show that $F$ contains an idempotent.

For a fixed element $a \in F$, let $A=\{x \in F \mid x a=a\}$. Then $A$ is nonvoid and $0<\mu(A)=\mu(\{a\})<\infty$. Note also that if $x \in A$ and 
$y \in A x^{-1} \cap F$ then $y a=y(x a)=(y x) a=a$, and so $y \in A$. Thus $A x^{-1} \cap F \subset A$ for all $x \in A$ and it follows that the restriction of $\mu$ to $A$ is an $r^{*}$-invariant measure on $A$. An application of the Recurrence Theorem (see Halmos, Lectures in ergodic theory, Chelsea, New York, 1956 ; p. 10) shows that each element of $A$ must have finite order. We complete the proof by recalling that each finite semigroup contains an idempotent.

\section{REFERENCES}

1. L. Argabright, Invariant means on topological semigroups, Pacific J. Math. 16 (1966), 1-11.

2. N. Bourbaki, Élements de mathématique. XIII, Livre VI: Integration, Chapters I-IV, Actualités Sci. Indust., Hermann, Paris, 1952.

3. A. H. Clifford, Semigroups containing minimal ideals, Amer. J. Math. 70 (1948), 521-526.

4. A. H. Clifford and G. B. Preston, The algebraic theory of semigroups, Math Surveys No. 7, Amer. Math. Soc., Providence, R. I., 1961.

5. R. Ellis, A note on continuity of the inverse, Proc. Amer. Math. Soc. 8 (1957), $372-373$.

6. E. Hewitt and K. Ross, Abstract harmonic analysis. I, Springer, Berlin, 1963.

7. J. H. Michael, Right invariant integrals on locally compact semigroups, J. Austral. Math. Soc. 4 (1964), 273-286.

8. P. S. Mostert, Comments on the preceding paper of Michael's, J. Austral. Math. Soc. 4 (1964), 287-288.

9. K. Numakura, On bicompact semigroups, Math. J. Okayama Univ. 1 (1952), 99-108.

10. W. G. Rosen, On invariant means over compact semigroups, Proc. Amer. Math. Soc. 7 (1956), 1076-1082.

UNIVERSity of CALIFoRNia, BERKELEY 